\title{
Ethische Fallbeschreibungen in der Schweizerischen Ärztezeitung
}

Medizin wirft, wo sie an ihre Grenzen stösst, ethische Fragen auf, die schwierig und meist nicht eindeutig zu beantworten sind. Medizinerinnen, die stattdessen klare Antworten bräuchten, sind oft von Ethikern enttäuscht und geneigt zu denken, Beschäftigung mit Ethik sei wenig hilfreich und eher etwas für Theoretikerinnen. Letztlich stellt sich die Frage, ob die vielbeschworene Zusammenarbeit zwischen Medizin und Ethik über blosse Berührungspunkte hinausgehen kann und zu gegenseitiger Durchdringung unterschiedlicher Denkweisen führt, oder ob sich lediglich eine pragmatische Arbeitsteilung der Disziplinen zwecks Abwicklung der Tagesgeschäfte ergibt.

Die Redaktion «Ethik» möchte einen Beitrag leisten, eine noch bei vielen Ärztinnen anzutreffende Skepsis gegenüber Ethik zu mildern und den Zugang zum ethischen Diskurs zu erleichtern. Dazu sollen unter der Rubrik «Ethik» in loser Folge Beiträge veröffentlicht werden, die anhand von Situationen aus dem klinischen Alltag ethisch interessante Fragestellungen illustrieren. Dazu wird die Redaktion mehrere Kommentare von Expertinnen - Ethikverständige im weiteren Sinn - einholen, welche diese Situation aus verschiedenen Perspektiven kommentieren und zu analysieren versuchen. Mit diesem Vorgehen soll verdeutlicht werden, dass unterschiedliche Herangehensweisen nicht nur möglich, sondern auch sinnvoll sein können und dass eine ethische Stellungnahme immer auch aus einer bestimmten Perspektive heraus geschieht. Mit der Publikation solcher Beiträge hoffen wir, dass die Leser nach und nach Verständnis dafür entwickeln, was Ethik sei, wozu sie diene und wozu nicht. Würden wir mit diesen Publikationen sogar einen kleinen Beitrag dazu leisten, dass sich vermehrt Ärzte aktiv am ethischen Diskurs beteiligen, wäre die vielleicht ehrgeizigste Zielsetzung erreicht.

Die Redaktion «Ethik» eröffnet diese Artikelserie mit einem Beitrag von G. Ebner vom Psychiatriezentrum Schaffhausen mit dem Titel «Ethik im Gesundheitswesen - Fall einer psychisch Kranken mit einer unheilbar körperlichen Krankheit mit Sterbewunsch» (Seite 2421). Ebner schildert den Fall einer wegen eines Karzinoms zunehmend pflegebedürftigen Frau, die auf einer psychiatrischen Pflegeabteilung behandelt wird. Diese Frau möchte ihrem Leiden durch Suizid ein Ende setzen und bittet um Beihilfe. Ebner legt die
Standpunkte der involvierten Ärzte und Pflegepersonen dar und erklärt den Weg bis zur Entscheidungsfindung.

Als Kommentatoren konnten Ruth BaumannHölzle als Theologin und Ethikerin, B. Küchenhoff und D. Hell als Psychiater sowie G. Bosshard als Rechtsmediziner gewonnen werden. Ruth Baumann-Hölzle zeigt, wie in diesem Fall der Entscheidungsprozess strukturiert werden kann, ohne den Entscheid selbst vorwegzunehmen; dabei erläutert sie die relevanten ethischen Begriffe. Die Psychiater und der Rechtsmediziner begründen in prägnanter Art und Weise ihre jeweiligen Standpunkte. Jede Stellungnahme ist in sich überzeugend, und doch wird erst durch ihre Gegenüberstellung die Spannbreite der Standpunkte bewusst, die in einer konkreten klinischen Situation möglich ist. Gerade das Fehlen eines endgültigen Kommentars macht die Faszination, die von einem immer wieder von neuem ansetzenden ethischen Diskurs ausgeht, sichtbar und regt hoffentlich auch den Leser zur eigenen Reflexion an.

Vor allem hoffen wir, dass durch diese ersten Beiträge die Idee des neuen Konzeptes anschaulich wird. Wir möchten die Leserinnen einladen, der Redaktion eigene Fallbeschreibungen, die sich für eine ähnliche Diskussion eignen könnten, einzureichen. Bei diesen Fallbeschreibungen kommt es weniger darauf an, dass bereits ein elaborierter ethischer Diskurs stattgefunden hat. Vielmehr sollte aufgrund einer auf das Wesentliche beschränkten und präzisen Schilderung die zur Diskussion stehende klinische Situation und die in ihr aufgetretenen ethischen Probleme auch für Dritte nachvollziehbar sein und einen Kommentar erlauben. Wir bitten aber, nur abgeschlossene Fälle einzureichen, da die Redaktion in keinem Fall die Rolle eines beratenden Gremiums, ja einer Ethikkommission übernehmen kann und will.

Für weitere Anregungen und Kritiken sind und bleiben wir offen.

Markus Trutmann, Christina aus der Au, Lazare Benaroyo, Christoph Rehmann-Sutter

PS: Auch für diese Beiträge gelten die Autorenrichtlinien der SÄZ, die auf der Website (www.saez.ch) konsultiert werden können. Als Maximalumfang für Beiträge gilt ein Richtwert von 10000 Zeichen. 